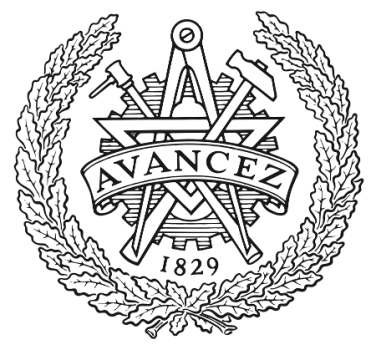

CHALMERS

UNIVERSITY OF TECHNOLOGY

\title{
Interphase Design of Cellulose Nanocrystals/Poly(hydroxybutyrate- ran-valerate) Bionanocomposites for Mechanical and Thermal Properties
}

Downloaded from: https://research.chalmers.se, 2023-04-26 13:05 UTC

Citation for the original published paper (version of record):

Magnani, C., Idström, A., Nordstierna, L. et al (2020). Interphase Design of Cellulose

Nanocrystals/Poly(hydroxybutyrate- ran-valerate)

Bionanocomposites for Mechanical and Thermal Properties Tuning. Biomacromolecules, 21(5):

1892-1901. http://dx.doi.org/10.1021/acs.biomac.9b01760

N.B. When citing this work, cite the original published paper. 


\title{
Interphase Design of Cellulose Nanocrystals/Poly(hydroxybutyrate- ran-valerate) Bionanocomposites for Mechanical and Thermal Properties Tuning
}

\author{
Chiara Magnani, Alexander Idström, Lars Nordstierna, Alejandro J. Müller, Philippe Dubois, \\ Jean-Marie Raquez, and Giada Lo Re*
}

Cite This: Biomacromolecules 2020, 21, 1892-1901

Read Online

ABSTRACT: Poly[(3-hydroxybutyrate)-ran-(3-hydroxyvalerate)] (PHBV) is a bacterial polyester with a strong potential as a substitute for oil-based thermoplastics due to its biodegradability and renewability. However, its inherent slow crystallization rate limits its thermomechanical properties and therefore its applications. In this work, surface-modified cellulose nanocrystals (CNCs) have been investigated as green and biosourced nucleating and reinforcing agent for PHBV matrix. Different ester moieties from the CNCs were thereby produced through a green one-pot hydrolysis/Fisher esterification. Beyond the improved dispersion, the CNCs surface esterification affected the thermal and

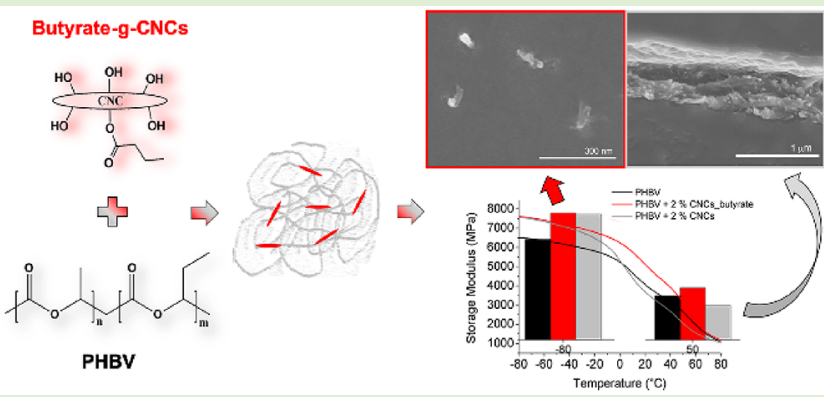
thermomechanical properties of PHBV. The results demonstrate that butyrate-modified CNCs, mimicking the PHBV chemical structure, brought a considerable improvement toward the CNCs/matrix interface, leading to an enhancement of the PHBV thermomechanical properties via a more efficient stress transfer, especially above its glass transition.

\section{INTRODUCTION}

The problem concerning the environmental impact of plastic pollution and waste management ${ }^{1,2}$ can no longer be neglected. As a consequence, single-use plastics have been recently banned ${ }^{3}$ in Europe, and the replacement of traditional not biodegradable oil-based polymers is becoming urgent. Thus, the interest in biosourced and particularly biodegradable materials is being increased, especially for their potential in packaging and disposable items, ${ }^{4}$ bringing them to the attention of benchmark commodity suppliers. Among other commercially available emerging polyesters, poly(3-hydroxybutyrate-ran-3-hydroxyvalerate) (PHBV) is a semicrystalline biosourced poly hydroxyalkanoate naturally produced by bacteria. ${ }^{5}$ Beyond its renewability ${ }^{6}$ and biodegradability, ${ }^{7-9}$ PHBV has many advantages compared to the well-known homopolymer poly(3-hydroxybutyrate) (PHB). Its mechanical and thermal properties can be readily tuned up by varying the relative percentage of the comonomer 3-hydroxyvalerate $(\mathrm{HV}) .{ }^{10}$ In this approach, a higher content of $\mathrm{HV}$ as comonomer is used to overcome the inherent brittleness of $\mathrm{PHB}$, although progressively lowering its crystallization rate. Commercially available grades of PHBV generally contain low HV content ( $<12$ wt \%). Therefore, PHBV application is limited because of its low ductility, high cost, ${ }^{11,12}$ and low thermal stability, ${ }^{13}$ as well as slow crystallization rate. ${ }^{14}$ Different approaches have been explored to overcome PHBV drawbacks, beyond the HV comonomer content, as blending with other polymers, ${ }^{15-17}$ addition of plasticizers, ${ }^{18-20}$ or nucleating agents. ${ }^{21}$ The use of nucleating agents is an economically viable approach already demonstrated for improving other commercialy available PHAs. ${ }^{22}$

Cellulose nanocrystals (CNCs) are of interest as emerging nucleating agents because of their natural origin and good mechanical properties exploitable to efficiently reinforce a polymer matrix. ${ }^{23} \mathrm{~A}$ few studies have reported a positive effect of CNCs, obtained from sulfuric or hydrochloric acid hydrolysis, as a nucleating agent for PHBV, leading to an amelioration of its mechanical properties, even at low nanofiller content. $^{24-29}$

An efficient reinforcement and a faster crystallization require a high level of dispersibility and individualization of CNCs, which remain a challenge. ${ }^{30,31}$ Their inherent high hydrophilic surface adversely interacts with the generally more hydrophobic polymer matrices, leading to agglomerate formation and depletion at the interphase, ${ }^{32}$ as already seen for CNCs in PHBV matrix by Malmir et al. ${ }^{26}$ Therefore, surface

Special Issue: Anselme Payen Award Special Issue

Received: December 20, 2019

Revised: February 19, 2020

Published: February 20, 2020

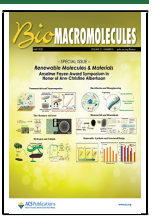



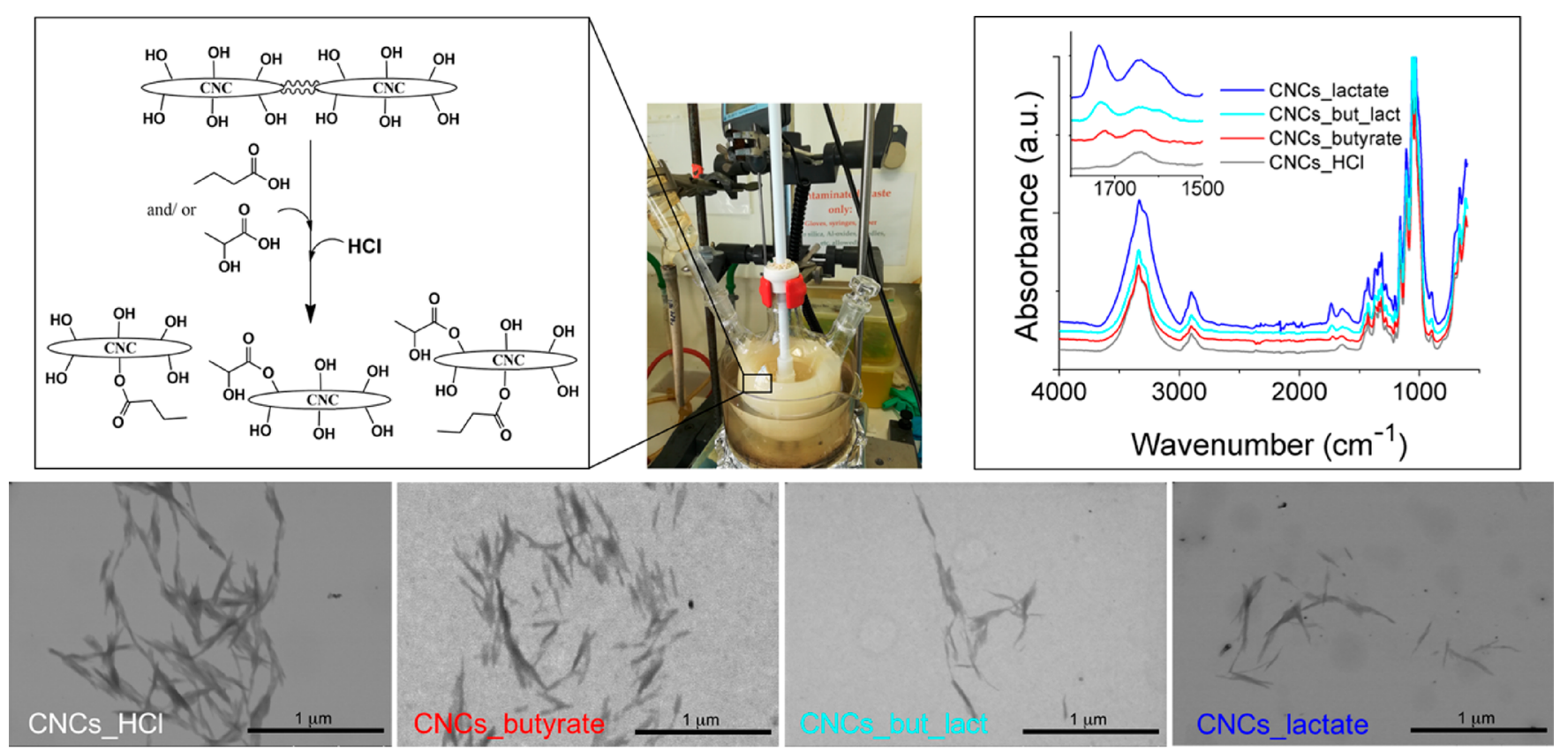

Figure 1. One-pot acid hydrolysis/Fischer esterification method (upper left image); FTIR spectra (upper right graph) and STEM micrographs of unmodified (CNCs_HCl) and modified nanocrystals with different ester moieties (CNCs_butyrate, CNCs_lactate, CNCs_but_lact, bottom image). A similar aspect ratio $(13.5 \pm 1.5)$ was calculated for the different CNCs from the morphological analysis on 100 different individualized CNCs. Samples observed in the STEM were directly obtained after the purification step following the synthesis (in water suspention $\approx 1.5$ wt $\%$ ).

modification has been the most exploited strategy to tackle this challenge. ${ }^{33,34}$ However, the main drawback associated with this approach lies in the addition of cost and time-consuming steps, which often require the use of not sustainable organic solvents and harmful chemicals. ${ }^{35,36}$

Braun et al. ${ }^{37}$ introduced a one-pot acid hydrolysis/Fischer esterification in water dispersion. This approach allowed for the CNCs preparation and their simultaneous surface esterification in a green and fast process, with high potential for scaling up. This method was found to be efficient with many acids. ${ }^{25,31,38,39} \mathrm{Yu}$ et al. used this method to graft organic acids carrying different content of hydroxyl functionalities and studied the influence of hydrogen bonds at the PHBV/CNCs interphase on mechanical properties of PHBV. ${ }^{31}$ Their results demonstrated in the case of PHBV bionanocomposites at 10 wt \% CNCs that the higher the hydrogen bonding content, the higher the Young's modulus. They also demonstrated a favorable effect on thermal stability of hydrogen bonds formed between the surface polyol moieties of CNCs and PHBV carboxyl groups. ${ }^{25}$

To our knowledge, no other studies have investigated the influence of different CNCs surface moieties on the PHBV thermal and dynamical-mechanical properties. In the current study, organic moieties with chemical structure similar to PHBV were selected to decorate the CNCs surface in order to improve the interaction with the polyester in a selective manner. This approach has been considered under the statement that an improved interface $\mathrm{CNCs} / \mathrm{PHBV}$ would promote the adhesion of the polyester to the CNCs surface leading to a more efficient stress transfer. ${ }^{40}$ This approach was found successful in the case of polylactide matrix by Spinella et al. $^{38}$ In this study, lactate decorated CNCs mimicking the backbone structure of polylactide significantly improved the thermal stability of the cellulose nanocrystals and the final performance of the bionanocomposite.
Green one-pot acid hydrolysis/Fischer esterification has herein been chosen to produce CNCs decorated with lactate and butyrate surface moieties. The reasons behind the choice of these two moieties lie on the assessment of the synergic or competitive effect of an improved thermal stability (provided by lactate moieties) and a mimicking of the PHBV molecular structure (with butyrate moieties). The present work focuses on the CNC/PHBV interface and its effect on mechanical and thermal properties. The rationale behind the bionanocomposite preparation method intended to limit the variables under investigation only to the surface chemistry at the interface. Aware of the substantial impact on the "green" aspect of the work, the bionanocomposites were therefore prepared through solvent casting for the sake of achieving a near-perfect dispersion. ${ }^{41,42}$ Indeed, this method prevents the CNCs aggregation, taking advantage of the mobility of the polymer chains dissolved in an organic solvent, while avoiding possible thermal degradation during melt processing.

The results highlighted that different modifications of CNCs affect the thermal behavior of PHBV and that butyrate moieties from the nanocellulose mimicking the polyester backbone lead to an improved CNCs/PHBV interphase and a more efficient stress transfer.

\section{EXPERIMENTAL SECTION}

Materials. PHBV (Enmat Y1000, $M_{\mathrm{w}}=2.6 \times 10^{5} \mathrm{~g} / \mathrm{mol}$ ) was produced by Tianan Biologic Material Co. Ltd. (Ningbo, China) and supplied by helianPolymer. Hydroxyvalerate (HV) content was indicated to be around $2.56 \mathrm{~mol} \%$. We calculated it as $0.8 \%$ from ${ }^{1} \mathrm{H}$ NMR. Ramie cellulose fibers were purchased from the Woolery (http://www.woolery.com/) and purified according to the protocol below. Butyric acid, lactic acid, hydrochloric acid (37\%), $\mathrm{NaOH}$, chloroform, and tetrachloroethane were obtained from VWR and used as received.

Cellulose Fibers Purification. $\mathrm{NaOH}$ treatment: $80 \mathrm{~g}$ of Ramie cellulose fibers were purified from impurities and residual hemicellulose through basic treatment. Briefly, fibers were soaked twice in 
a $\mathrm{NaOH}$ solution $\left(4 \mathrm{wt} \%\right.$ ), first at $80{ }^{\circ} \mathrm{C}$ for $2 \mathrm{~h}$ and then at $60{ }^{\circ} \mathrm{C}$ overnight, under mechanical agitation, washing them with deionized water between the two treatments. Bleached fibers were then rinsed with deionized water until a neutral $\mathrm{pH}$ and dried under vacuum at 30 ${ }^{\circ} \mathrm{C}$.

Cellulose Nanocrystal Preparation and Functionalization: One-Pot Acid Hydrolysis/Fischer Esterification. A total of $10 \mathrm{~g}$ of fibers were soaked overnight in the organic acid chosen (lactic or butyric acid). The preparation was carried out at $105{ }^{\circ} \mathrm{C}$ under mechanical stirring, adding in a row of water and $\mathrm{HCl}$ (Figure 1). $\mathrm{HCl}$ concentration was $0.05 \mathrm{M}$ for CNCs_butyrate and $0.07 \mathrm{M}$ for CNCs_lactate. The final solution contained $90 \mathrm{wt} \%$ of acid compared to water. The reaction was quenched after $9 \mathrm{~h}$, reaching $\mathrm{pH}=5$ through water rinses (centrifuge $4000 \mathrm{rpm}, 5 \mathrm{~min}, 10^{\circ} \mathrm{C}$ ). To recover cellulose nanocrystals, the suspension was mixed for $20 \mathrm{~min}$ with an immersion mixer, and subsequently, the opalescent supernatants containing nanocrystals were collected from the following centrifugations and combined to obtain the final water suspension (STEM). Depending on the characterization, a certain amount of suspension was freeze-dried (FT-IR, XRD, TGA, as specified in the Characterization section).

Control Nanocrystals. A total of $10 \mathrm{~g}$ of bleached fibers have been soaked in water for one night. The temperature has been increased to $105{ }^{\circ} \mathrm{C}$ under reflux and mechanical stirring. Then, $\mathrm{HCl}$ $37 \%$ has been added slowly to reach a concentration of $2.5 \mathrm{M}$, and the hydrolysis has been quenched after $1 \mathrm{~h}$ by adding DI ice cubes and with the help of an ice bath. The rest of the protocol was the same as for functionalized nanocrystals.

Bionanocomposites Solvent Casting. To boost CNCs dispersion (after the synthesis in water) in the polymer matrix, preventing the irreversible hydrogen bonds formation upon drying (so their aggregation) and degradation during melt processing, CNCs were transferred from water to chloroform through solvent exchange, gently increasing the hydrophobicity of the solvent. In particular, the solvent sequence was water, ethanol, acetone, and finally, chloroform, and their stability was assessed (Supporting Information). PHBV was dissolved in a mixture of chloroform and tetrachloroethane (50:50) for at least $3 \mathrm{~h}$ at $70{ }^{\circ} \mathrm{C}$. For each composite, the desired amount of CNCs suspension in chloroform was added to a PHBV solution. The mix was then homogenized through mechanical stirring and sonication. Then it was poured in a glass Petri dish, and a casted film was obtained in an oven at $50{ }^{\circ} \mathrm{C}$, controlling the decreasing of the pressure until 0 mbar to avoid bubble formation. Films were then dried at $50{ }^{\circ} \mathrm{C}$ for one night. CNCs wt $\%$ was evaluated considering negligible the weight changes due to the surface modification.

Samples for Mechanical Tests. Bars with an average dimension of $2.5 \mathrm{~cm} \times 0.5 \mathrm{~cm} \times 0.45 \mathrm{~mm}$ were obtained from the casted films through compression molding at $180{ }^{\circ} \mathrm{C}$.

Characterization. Attenuated total reflectance Fourier transform infrared spectroscopy (FT-IR) was carried out on freeze-dried CNCs using a Bruker Tensor 17 spectrometer. Ultraviolet-visible spectroscopy (UV-vis) was performed on the bionanocomposite films using a spectrophotometer Shimadzu UV-2600 in the wavelength range between 200 and $800 \mathrm{~nm}$. Scanning electron microscopy (SEM) was carried out with the apparatus Hitachi SU8020. CNC morphology was observed in the transmission mode (STEM) on a water suspension of CNCs, recovered after a purification step following the synthesis, and the aspect ratio was calculated from the morphological analysis of 100 different individualized CNCs (the scattering of the data was lower than 5\%). Morphological analysis of the bionanocomposites was carried out on a DMTA specimen crosssection obtained by cryofracture in liquid nitrogen. The X-ray diffractometry (XRD) was performed on freeze-dried CNCs by a Panalytical Empirean diffractometer with an area detector operating under $\mathrm{Cu} \mathrm{K} \alpha(1.5418 \AA)$ radiation $(40 \mathrm{kV}, 40 \mathrm{~mA})$. The crystallinity was assessed as the areas of the crystalline diffraction peaks to the total area under the curve between $2 \theta=10$ and $50^{\circ}$. The crystallinity of CNCs and bionanocomposites were determined based on the Rietveld-Ruland approach: ${ }^{4344}$

$$
X_{\mathrm{XRD}}=\frac{A_{\mathrm{CR}}}{A_{\mathrm{CR}}+A_{\mathrm{am}}} \times 100 \%
$$

where $A_{\mathrm{CR}}$ is the area for crystalline peaks and $A_{\mathrm{am}}$ is area for amorphous peaks. The crystallite sizes of 002 lattice planes were estimated by using the Scherrer equation: ${ }^{24,28}$

$$
D_{h k l}=\frac{0.9 \lambda}{B_{h k l} \cos \theta}
$$

where $D_{h k l}$ is the crystallite size in the direction normal to the $h k l$ lattice planes, $\lambda$ is the radiation wavelength $(1.54 \AA)$, and $B_{h k l}$ is the full width at half-maximum in radians of the corresponding $h k l$ lattice planes.

Solid-state cross-polarization magic angle spinning carbon-13 nuclear magnetic resonance (CP/MAS ${ }^{13} \mathrm{C}$ NMR) was evaluated using a single pulse direct polarization experiment on freeze-dried CNCs, and quantitative data of the modifications could be obtained. Comparing the integral of the lactic acid signal $\mathrm{L} 3$ at $18 \mathrm{ppm}$ with the total integral of the $\mathrm{C} 4$ signal of the $\mathrm{CNCs}$, the lactic acid modification degree of substitution, DS, could be obtained. Similarly, comparing the average integral of the butyric acid signals B2, B3, and $\mathrm{B} 4$, with the $\mathrm{C} 4$ signal of the CNCs, the butyric acid DS could be obtained. For the samples containing both lactic and butyric acid some overlap of the modification signals was inevitable. To circumvent this, similarly to above but instead only using the well resolved signals B2 and B4, the DS for the butyric acid could be obtained. No lactic acid signal could be integrated without severe overlap from other signals. However, estimating the B3 integral (overlapping with L3) as the average of the integrals for B2 and B4 and then removing this from the combined integral of B3 and L3, the L3 integral could be estimated, hence, giving the lactic acid DS. The obtained DS for all modifications could be seen in Table 1. By

Table 1. Crystallinity Index $\left(\chi_{\mathrm{NMR}}\right)$ and Degree of Substitution (DS) for All Samples ${ }^{a}$

$\begin{array}{lccccc} & \begin{array}{c}\chi_{\text {NMR }} \\ (\%)\end{array} & \begin{array}{c}\text { DS (\%) } \\ \text { lactate }\end{array} & \begin{array}{c}\text { DS (\%) } \\ \text { butyrate }\end{array} & \begin{array}{c}\mathrm{DS}_{\text {surf }}(\%) \\ \text { lactate }\end{array} & \begin{array}{c}\mathrm{DS}_{\text {surf }}(\%) \\ \text { butyrate }\end{array} \\ \text { CNCs_HCl } & 63 & & & & \\ \text { CNCs_lactate } & 59 & 3.3 & & 29 & \\ \text { CNCs_butyrate } & 58 & & 1.8 & & 15 \\ \text { CNCs_but_lact } & 58 & 2.1 & 1.6 & 16 & 13\end{array}$

${ }^{a}$ For lactic acid modification, DS values were obtained comparing the integral of the lactic acid signal L3 at $18 \mathrm{ppm}$ with the total integral of the C4 signal of the CNC. For the butyric acid, DS values were obtained comparing the average integral of the butyric acid signals B2, $\mathrm{B} 3$, and B4, at 32, 15, and $10 \mathrm{ppm}$, respectively, with the C4 signal of the CNC. The assessed relative amount of accessible surface of about $11 \%$, enabled the estimation of the degree of substitution on the surface, $\mathrm{DS}_{\text {surf }}$.

studying the CNCs in the wet state, NMR signals corresponding to the accessible surfaces of the CNCs can be distinguished from those on internal inaccessible surfaces. ${ }^{45}$ The analysis provided a relative amount of accessible surface signals to be around $11 \%$ for all modified CNCs. Knowing the relative amount of accessible surface, the degree of substitution on the surface, $\mathrm{DS}_{\text {surf }}$, could be calculated for all modified CNC samples, as seen in Table 1. Thermogravimetrical analysis was carried out on freeze-dried CNCs and casted films with a TGA Q500 apparatus from T.A. Instruments under a nitrogen flow from r.t. to $800{ }^{\circ} \mathrm{C}$ with a step of $20{ }^{\circ} \mathrm{C} / \mathrm{min}$. Casted films were analyzed by differential scanning calorimetry (DSC) on a DSC Q2000 apparatus, calibrated with indium from T.A. using aluminum pans and nitrogen atmosphere. Nonisothermal analysis were performed following a heat-cool-heat program from -80 to $190{ }^{\circ} \mathrm{C}$ (rate 20 ${ }^{\circ} \mathrm{C} / \mathrm{min}$ ), with an isotherm of $3 \mathrm{~min}$ at $190{ }^{\circ} \mathrm{C}$. Isothermal analysis were performed at $120^{\circ} \mathrm{C}$ after an heating ramp until $190{ }^{\circ} \mathrm{C}$ with 


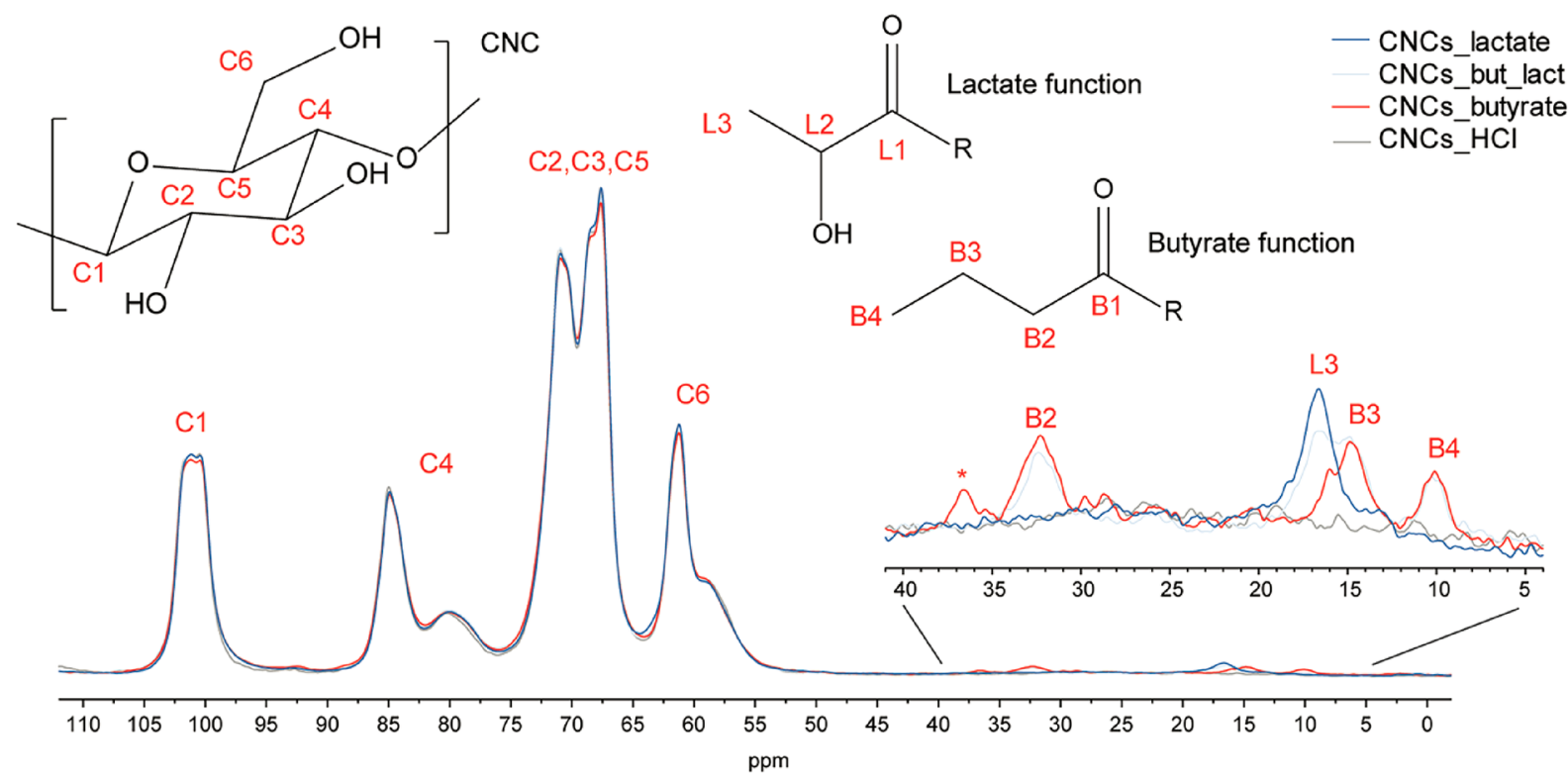

Figure 2. $\mathrm{CP} / \mathrm{MAS}{ }^{13} \mathrm{C}$ NMR spectrum of $\mathrm{CNCs} \_\mathrm{HCl}, \mathrm{CNCs}$ lactate, CNCs_butyrate, and CNCs_but_lact. Zoom-in of the spectra highlight some of the signals from the modifications. Inset shows the structural formula of the anhydroglucose unit of the CNCs as well as the lactic and butyric acid modifications, with numbered carbons.

isotherm of $3 \mathrm{~min}$ and cooling at $60^{\circ} \mathrm{C} / \mathrm{min}$. The crystallinity $\chi_{\mathrm{DSC}}$ of PHBV bionanocomposites was calculated as follows:

$$
X_{\mathrm{DSC}}=\frac{\Delta H_{\mathrm{m}}}{\left(1-w_{(\mathrm{CNCs})}\right) \Delta H_{100}} \times 100 \%
$$

where the $w_{(\mathrm{CNCs})}$ is the mass content of CNCs, $\Delta H_{\mathrm{m}}$ is the measured fusion enthalpy, and $\Delta H_{100}$ is chosen as $146.6 \mathrm{~J} / \mathrm{g}$ for theoretical fusion enthalpy of $100 \%$ crystalline PHBV due to low HV content of PHBV. $^{31}$

Half-crystallization time was calculated from isothermal analysis as the time to reach $50 \%$ of crystallization peak area. Mechanical performance ofthe bionanocomposites was evaluated by dynamic mechanical thermal analysis (DMTA) on compression molded bars using a DMTA Q800 apparatus (T.A. Instrument) equipped with a single cantilever clamp, in frequency sweep $\left(0.1-10 \mathrm{~Hz}, 25^{\circ} \mathrm{C}\right.$, strain $0.02 \%)$ and temperature sweep $\left(-80\right.$ to $100{ }^{\circ} \mathrm{C}, 2{ }^{\circ} \mathrm{C} / \mathrm{min}, 1 \mathrm{~Hz}$, strain $0.02 \%$ ).

\section{RESULTS AND DISCUSSION}

In order to elaborate PHBV-based bionanocomposites, we propose to surface-modify cellulose nanocrystals using a onepot acid hydrolysis/Fischer esterification process from butyric acid, lactic acid, and their mixture, ${ }^{25,37,38}$ as schematically sketched in Figure 1. For sake of comparison, nonfunctional cellulose nanocrystals were obtained from $\mathrm{HCl}$-hydrolysis. In accordance with our previous studies, ${ }^{38,39}$ the morphology of these nanocrystals was similar to a typical needle shape of an average dimension of $260 \mathrm{~nm}$ in length and $20 \mathrm{~nm}$ in width (Figure 1). The presence of peak centered at around 1730 $\mathrm{cm}^{-1}$ in the FTIR spectra, corresponding to the ester $(\mathrm{C}=\mathrm{O})$ stretching, indicates that the Fisher esterification took place simultaneously during the acid hydrolysis. X-ray analyses (Figure S1) confirmed that the typical crystalline structure of cellulose I for the unmodified and modified nanocrystals was maintained after hydrolysis upon these characteristic peaks at $14.6^{\circ}, 16.3^{\circ}, 20.3^{\circ}$, and $34^{\circ}$. These correspond to the respective crystalline lattices $(1-10),(110),(200)$, and (400), confirming that the Fischer esterification represents a mild surface-modification method. ${ }^{38}$
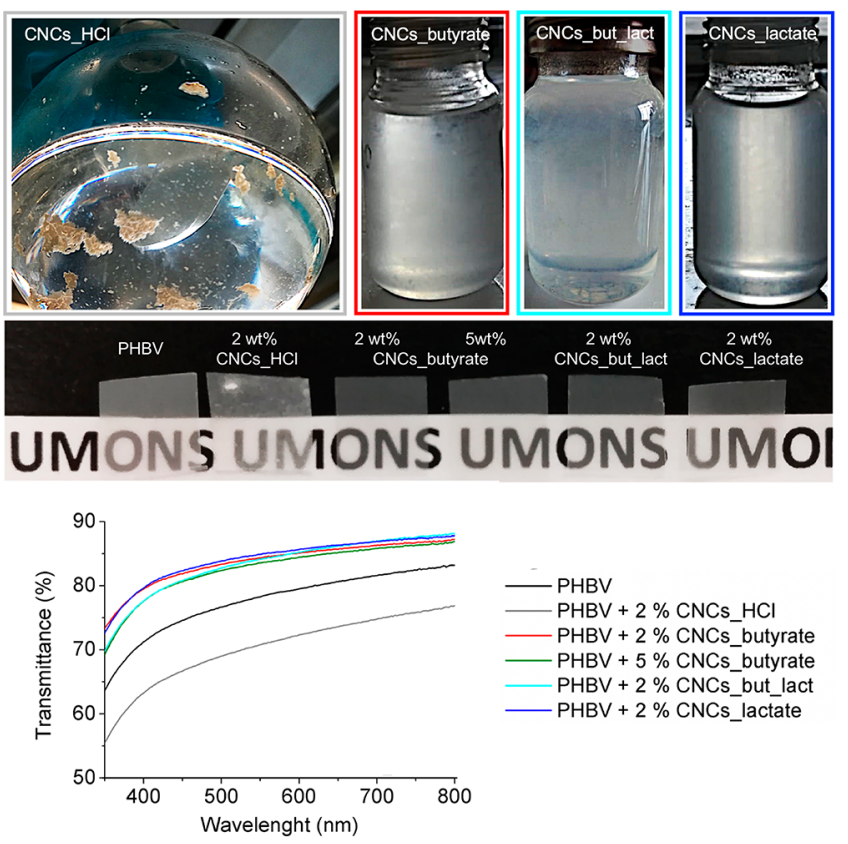

$-\mathrm{PHBV}$

- PHBV $+2 \% \mathrm{CNCs}$ HCl PHBV $+2 \%$ CNCs_butyrate PHBV $+5 \%$ CNCs_butyrate PHBV $+2 \%$ CNCs_but_lact PHBV $+2 \%$ CNCs_lactate

Figure 3. Dispersion in chloroform of the unmodified and modified cellulose nanocrystals after $15 \mathrm{~min}$ from the mechanical stirring (top image), visual aspect (central image), and UV-vis spectra (bottom image) of bionanocomposites with different amounts and types of CNCs.

The degree of surface substitution ( $\left.\mathrm{DS}_{\text {surf }}\right)$ compared to the bulk assessment (DS) and the crystallinity extent $\left(\chi_{\mathrm{NMR}}\right)$ of modified and unmodified CNCs was then determined using $\mathrm{CP} / \mathrm{MAS}{ }^{13} \mathrm{C}$ NMR spectroscopy (Figure 2) and the values are reported in Table 1.

From the CP/MAS ${ }^{13} \mathrm{C}$ NMR spectrum, the surfacemodification from butyric and lactic acid was confirmed, following the peaks between 5 and 37 ppm (L3 and B2, B3, and B4), together with the typical peaks of cellulose nanocrystals. The carbonyl carbons, L1 and B1, are not 
Table 2. Thermal Results of Neat PHBV and Bionanocomposites at 2 wt $\%$ of Different $\mathrm{CNCs}^{a}$

\begin{tabular}{|c|c|c|c|c|c|c|c|}
\hline & $\Delta H_{\mathrm{m}}(\mathrm{J} / \mathrm{g})$ & $\chi_{\mathrm{DSC}}^{b}(\%)$ & $T_{\mathrm{c}}\left({ }^{\circ} \mathrm{C}\right)$ & $t_{1 / 2 @ 120^{\circ} \mathrm{C}^{b}}(\mathrm{~min})$ & $\chi_{\mathrm{XRD}}^{b}(\%)$ & $D_{121}^{d}(\mathrm{~nm})$ & $D_{002}{ }^{d}(\mathrm{~nm})$ \\
\hline PHBV & 88 & 47 & 99 & 11 & 56 & 8.5 & 5.5 \\
\hline $\mathrm{PHBV}+2 \%$ CNCs_butyrate & 99 & 50 & 103 & 4 & 56 & 7.7 & 4.3 \\
\hline PHBV + $2 \%$ CNCs_lactate & 88 & 46 & 98 & 5 & 46 & 8.7 & 5.9 \\
\hline PHBV $+2 \%$ CNCs_but_lact & 102 & 51 & 98 & 5 & 54 & 8.0 & 4.9 \\
\hline $\mathrm{PHBV}+2 \%$ CNCs_HCl & 83 & 44 & 99 & 12 & 53 & 8.0 & 5.1 \\
\hline
\end{tabular}

${ }^{a}$ From the second heating of non-isothermal DSC analysis was assessed $\Delta H_{\mathrm{m}}, \chi_{\mathrm{DSC}}$, and $T_{\mathrm{c}}$, corresponding, respectively, to melting enthalpy, crystallization temperature, and crystallinity. The half-crystallization time $t_{1 / 2\left(120^{\circ} \mathrm{C}\right)}$ has been estimated from the isothermal crystallization at 120 ${ }^{\circ} \mathrm{C}$. Crystallinity $\chi_{\mathrm{XRD}}$ and the dimension on the direction perpendicular to (121) and (002) crystal faces, $D_{121}$ and $D_{002}$, respectively, are calculated

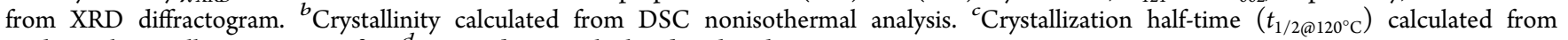
isothermal crystallization at $120^{\circ} \mathrm{C} .{ }^{d} D_{002}$ and $D_{121}$ calculated with Scherrer equation.
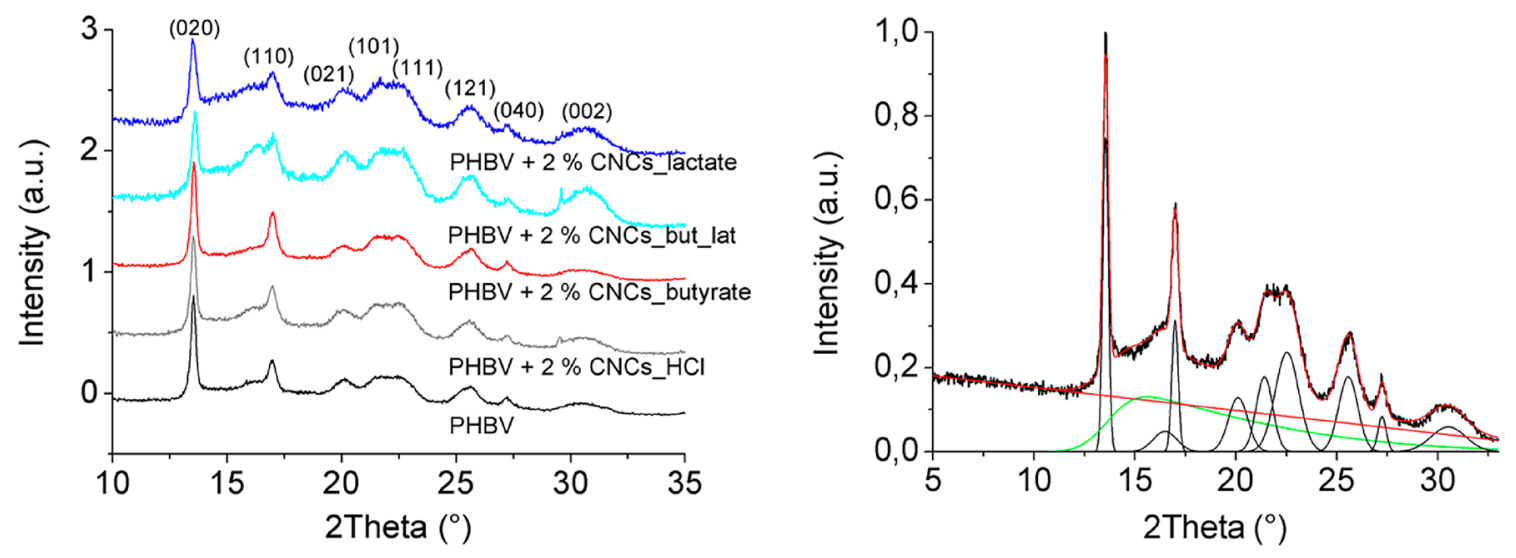

Figure 4. XRD diffractograms of neat PHBV and bionanocomposites at a content of 2 wt \% of CNCs with different surface chemistry (left); example of deconvolution of XRD spectra with PeakFit (bionanocomposites containing CNCs_butyrate, right).
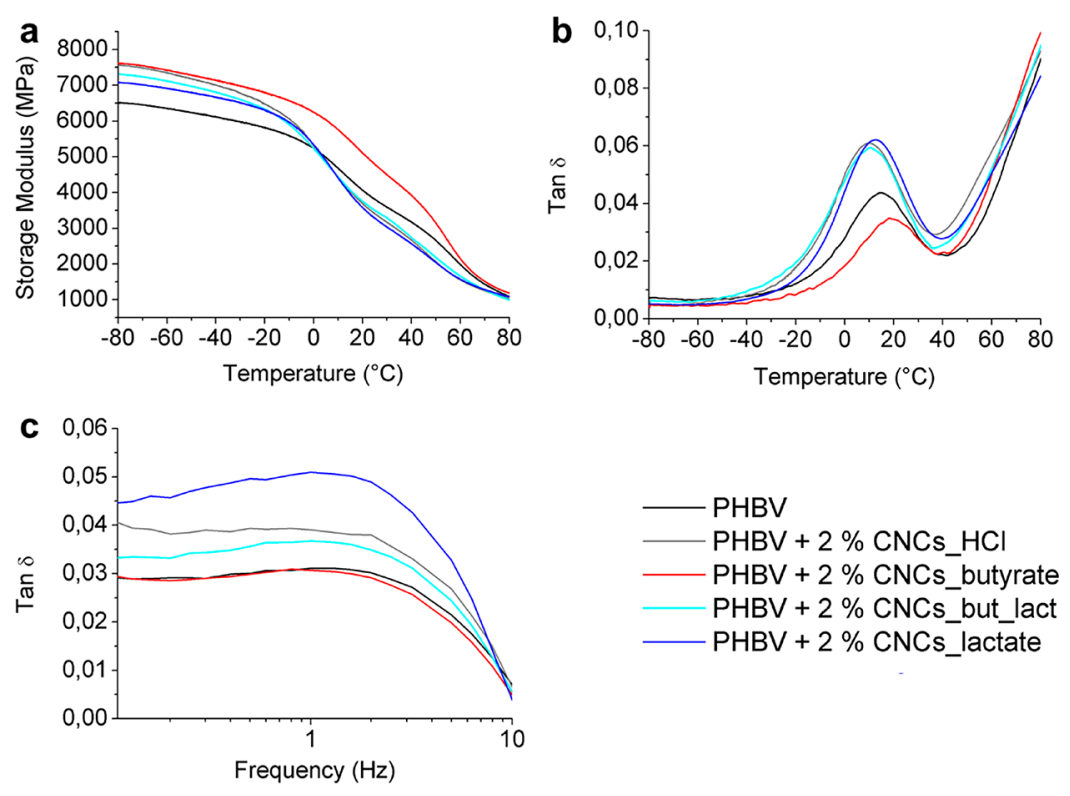

Figure 5. Dynamical-thermomechanical properties obtained from DMTA analysis of neat PHBV and bionanocomposites with CNCs with different surface chemistry; (a) Storage moduli and (b) $\tan \delta$ in temperature sweep analysis $\left(2{ }^{\circ} \mathrm{C} / \mathrm{min}, 1 \mathrm{~Hz}, 0.02 \%\right.$ strain); (c) $\tan \delta$ in frequency sweep $\left(25{ }^{\circ} \mathrm{C}, 0.02 \%\right.$ strain $)$.

probed effectively with the $\mathrm{CP} / \mathrm{MAS}$ pulse sequence hence not visible in the spectra (region not shown). In this work, the L2 carbon in the lactate modification was not assigned due to severe overlap with the large cellulose signals; however, the signals have previously been shown to appear around 70 ppm. ${ }^{46}$ For the CNCs_butyrate, a small signal at $37 \mathrm{ppm}$ (marked with $*$, Figure $\overline{2}$ ) originated from a residual solvent in the sample. The additional spectral features from the wet state analysis enabled the assessment of the accessible surface (about the 11\%), ${ }^{45,47}$ so allowing to distinguish the bulk from the surface degree of substitution (DS and $\mathrm{DS}_{\text {surf }}$, respectively), obtained using the $\mathrm{C} 4$ signal of the $\mathrm{CNCs}$ as the reference. For the CNCs_lactate nanocrystals, a degree of surface modification (DS) of $29 \%$ in lactate moieties was assessed, while a 
Table 3. Average DMTA Results of Neat PHBV and Bionanocomposites at 2 wt \% of Different CNC Types ${ }^{a}$

\begin{tabular}{|c|c|c|c|c|c|}
\hline & $\begin{array}{l}G_{\left(@-80^{\circ} \mathrm{C}\right.}^{\prime} \\
(\mathrm{MPa})\end{array}$ & $\begin{array}{l}G^{\prime} @ 25^{\circ} \mathrm{C} \\
(\mathrm{MPa})\end{array}$ & $\begin{array}{l}T_{\mathrm{g}}^{b} \\
\left({ }^{\circ} \mathrm{C}\right)\end{array}$ & $\begin{array}{l}T_{\alpha}^{b} \\
\left({ }^{\circ} \mathrm{C}\right)\end{array}$ & $\mathrm{DF}^{c}$ \\
\hline PHBV & 6504 & 3812 & 12 & 14 & 0.044 \\
\hline $\begin{array}{l}\text { PHBV }+ \\
2 \% \text { CNCs_butyrate }\end{array}$ & 7612 & 4788 & 17 & 19 & 0.035 \\
\hline $\begin{array}{l}\text { PHBV }+ \\
2 \% \text { CNCs_lactate }\end{array}$ & 7067 & 3283 & 8 & 13 & 0.063 \\
\hline $\begin{array}{l}\text { PHBV }+ \\
2 \% \text { CNCs_but_lact }\end{array}$ & 7309 & 3496 & 6 & 10 & 0.059 \\
\hline $\begin{array}{l}\mathrm{PHBV}+ \\
2 \% \mathrm{CNCs} \mathrm{HCl}\end{array}$ & 7550 & 3407 & 5 & 10 & 0.060 \\
\hline
\end{tabular}

${ }^{a}$ Scattering of the data below $3 \% .{ }^{b}$ Glass transition, $T_{g}$, was calculated from the Loss Modulus peak maximum obtained by a temperature sweep. ${ }^{c}$ Damping factor, DF, was calculated as $\tan \delta$ peak maximum, obtained by a temperature sweep.

DS of $15 \%$ was estimated for the butyrate ones. The higher acidity of lactid acid could explain its higher reactivity toward the Fisher esterification, although previous studies stated that there is no evident influence of acids strength on the substitution degree. ${ }^{37,39}$ For the simultaneous modification, the DS value of both lactic and butyric acid was of 15 and 13\%, being slightly lower than the corresponding CNCs lactate and CNCs_butyrate nanocrystals. This indicates that a certain competition between both acids can occur during the simultaneous surface decoration. The DS values calculated were in line with the relatively low degree of modification required to preserve the $\mathrm{CNCs}$ crystallinity, ${ }^{48}$ in accordance with the crystallinity index, $\chi_{\mathrm{NMR}}($ Table 1$)$. The $\chi_{\mathrm{NMR}}$ was calculated by comparing the crystalline and amorphous signals of the $\mathrm{C} 4$ carbon in the anhydroglucose unit of $\mathrm{CNCs}^{43,46}$ (Park, 2010). No significant changes in the crystallinity of the samples for the ester-decorated CNCs could be seen after modification, which is interesting toward reinforcing effect in cellulose-based nanocomposites. ${ }^{48}$ The surface modification slightly disturbs the structuring of the unmodified cellulose, as already observed in other studies. ${ }^{49,50}$ A slight decrease of the $\chi_{\mathrm{NMR}}$ for the CNC could be seen after modifications, in line with values obtained from previous work. ${ }^{46,51,52}$ Harsher modifications have also been shown to render larger changes in the CI values. ${ }^{53,54}$ XRD assessment of the crystallinity of the different CNCs showed values in line with the literature ${ }^{31}$ and confirmed the $\mathrm{CP} / \mathrm{MAS}{ }^{13} \mathrm{C}$ NMR analysis while presenting a higher absolute value, as already reported elsewhere. ${ }^{47}$

First attempts were then carried out to see whether these asmodified cellulose nanocrystals can be readily dispersed into PHBV by solvent-casting. Practically speaking and in order to circumvent the difficulty to redisperse freeze-dried CNCs in organic solvents, ${ }^{23,48}$ never-dried nanocrystals from solvent exchange to chloroform have been herein investigated, with the goal to obtain near-perfect bionanocomposites from solvent casting. Figure 3 illustrates the quality of the dispersion of $\mathrm{CNCs} \mathrm{HCl}$ and functionalized nanocrystals in chloroform. All the ester-modified CNCs showed a similar dispersibility (Figure S3) during the $15 \mathrm{~min}$, as an overestimated time needed for the mixing of the CNCs with the polymer solution. From the visual analysis, it is clear that the ester functionalities of the modified CNCs avoid the aggregation of these nanocrystals in chloroform in contrast to the unreversible agglomeration of the unmodified ones (CNCs_HCl, Figure 3).

The good stability in chloroform is a key factor to obtain a good dispersion of nanocrystals in a polymer solution during solvent casting. ${ }^{23,48}$ Therefore, a content of 2 wt \% in CNCs in the bionanocomposites was chosen to evaluate their transparency as casted films (Figure 3, bottom image) as a first evaluation of the near-perfect dispersion quality expected. A 5 wt \% CNCs butyrate-based nanocomposite was also prepared for comparison. The visual aspect of the films showed good transparency and homogeneity for the bionanocomposites containing functionalized CNCs, appearing more transparent than the film made of neat PHBV. In the presence of $\mathrm{CNCs} \mathrm{HCl}$ instead, an increased opalescence can be distinguished in the corresponding bionanocomposite film that can be explained, assuming the presence of micrometric agglomerates. $^{25}$ The improved dispersion in choroform, therefore, resulted in a uniform transparency of the modified CNCs-based bionanocomposite compared with the unmodified CNCs-based ones, as further confirmed by the UV-vis spectroscopy (Figure 3). The film containing the 2 wt \% of CNCs_HCl showed about a $20 \%$ decrease in transmittance compared to the neat matrix, while the modified CNCs led to an increase in transmittance of about $14 \%$. This result is in contrast with previous works, showing that the addition into chloroform PHBV solution of 1 wt \% of CNCs (modified with formic acid, eventually coupled with silver nanoparticles ${ }^{25,55}$ ) decreased the transparency of solvent-casted films.

Thermal properties of the bionanocomposites were investigated to assess the nucleating CNCs effect on PHBV (Figure S4 and Tables S3-S5). We must highlight that all modified CNCs showed an improvement in terms of the degradation temperature, especially for lactate-functionalized ones using thermogravimetric analysis under a nitrogen atmosphere (Figure S2). ${ }^{38}$ This enhanced thermostability was even noticed in the case of PHBV composites after $\mathrm{CNC}$ addition into PHBV, as already observed for similar CNC-based bionanocomposites produced by solvent casting ${ }^{25}$ (Figures S4 and S5). From Table 2, the bionanocomposites containing CNCs_butyrate showed an increased $T_{\mathrm{c}}\left(103{ }^{\circ} \mathrm{C}\right)$, compared to the neat PHBV and other bionanocomposites ( 98 and $99{ }^{\circ} \mathrm{C}$ ). This is consistent with a more pronounced nucleating effect of the CNCs decorated with butyrate moieties. A more effective nucleation is suggested by the half-crystallization time calculated from the isothermal analysis at $120{ }^{\circ} \mathrm{C}$ (Table 2), in the presence of CNCs butyrate, followed by the other modified CNCs. Indeed, neat PHBV $\tau_{1 / 2}$ was reduced by more than half in the presence of functionalized nanocrystals, and it was even lower in the case of CNCs butyrate. These results can be explained by an improved interface and/or an improved dispersion of ester modified CNCs into the PHBV matrix. ${ }^{56}$ On the contrary, CNCs_HCl seemed not to have any nucleating effect.

The bionanocomposites diffractograms present a typical crystalline peak pattern corresponding to PHBV lattice, ${ }^{57}$ further confirming that neither the crystalline characteristics of PHBV nor its average degree of crystallinity were affected by the surface modification (Figure 4 and Table 2). Interestingly, the presence of CNCs and their different surface chemistry seems to affect the crystal dimensions in the case of PHBV. The approximate dimensions were calculated in the direction perpendicular to two different crystalline lattice planes, (121) and (002), applying the Scherrer equation ${ }^{24,28}$ (Table 2).

To unravel the effect of the different surface esterification on the structure of PHBV crystals, X-ray diffraction was used.

Further crystallization kinetics studies are under investigation for deeper comprehension about the crystallization 


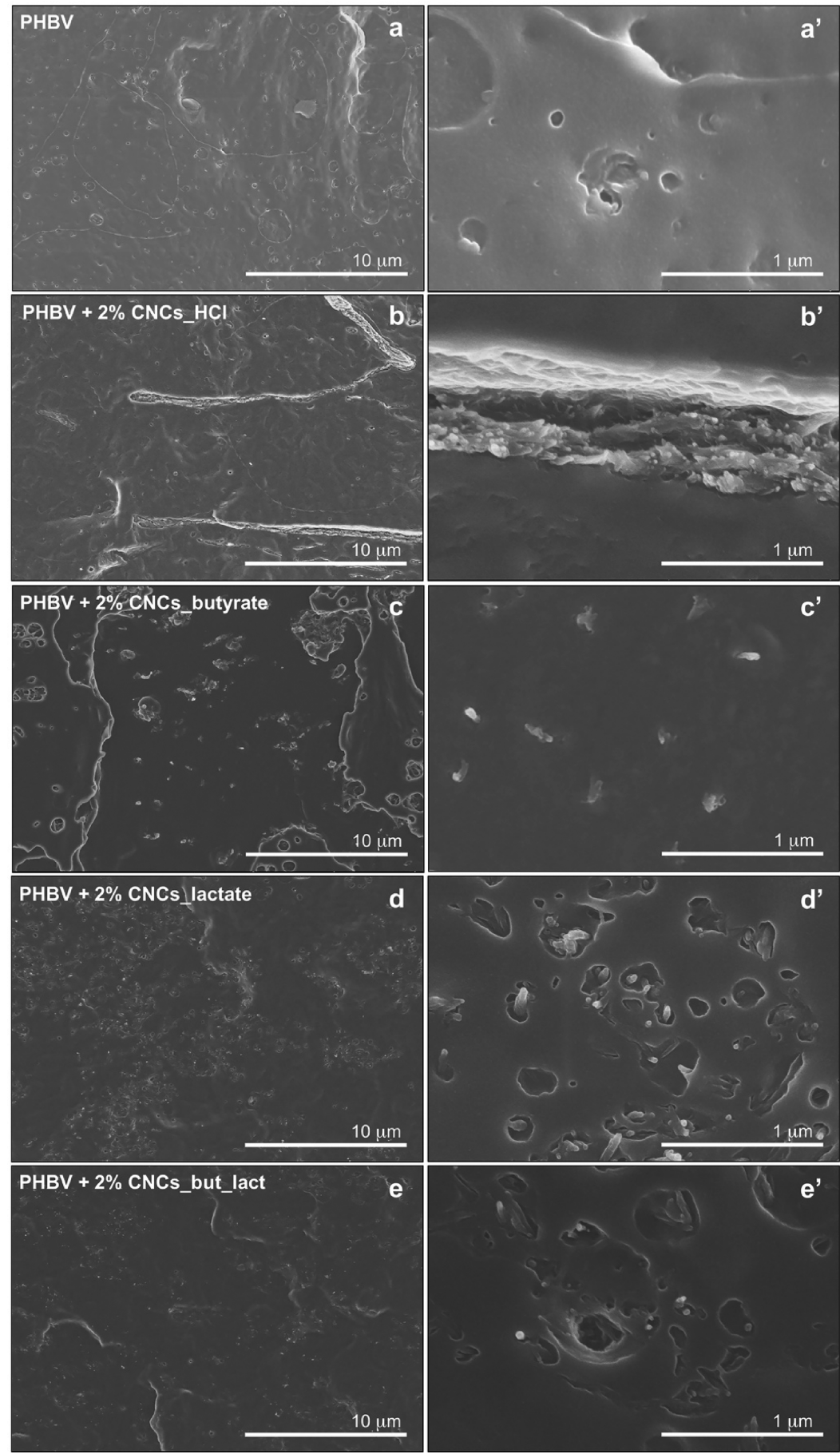

Figure 6. SEM micrographs after cryofracture of $\operatorname{PHBV}\left(a, a^{\prime}\right)$ and of the bionanocomposites at 2 wt \% content of differently surface decorated CNCs $\left(\mathrm{b}-\mathrm{e}^{\prime}\right)$ at two different magnifications (bars $10 \mu \mathrm{m}$, left micrographs, and $1 \mu \mathrm{m}$, right micrographs).

and nucleation phenomena occurring in these bionanocomposites, but they are beyond the scope of the current study.

Dynamical mechanical analysis (Figure 5) were carried out to further investigate an eventual correlation between the different surface features related with the different CNCs/ PHBV interface and their efficiency toward stress transfer.

Before the glass transition $\left(T<T_{\mathrm{g}}\right)$, the storage moduli increase for all the bionanocomposites, as a consequence of CNCs addition (Figure 5 a). The highest moduli at lower temperature are shown by the bionanocomposites with CNCs_HCl and CNCs_butyrate. The importance of the interphase came out across the $T_{\mathrm{g}}$ and over, that is the region where the reinforcing effect is more evident since an increasing mobility of the matrix is expected. In particular, across and after the glass transition dynamical mechanical behavior of CNCs_butyrate-based bionanocomposite exhibits a peculiar slow decreasing in the modulus. At $-80{ }^{\circ} \mathrm{C}$, its flexural storage modulus is $17 \%$ higher than the one of the neat PHBV. At room temperature, its modulus is still the $26 \%$ higher than the corresponding modulus of the neat PHBV. Moreover, among the bionanocomposites, this is the only one presenting a shift of $T_{\mathrm{g}}$ from 12 to $17{ }^{\circ} \mathrm{C}$. This is consistent with a more 
pronounced damping (Figure $5 \mathrm{~b}$ and Table 3 ) that indicates a hindered molecular mobility of the matrix at the molecular level, while the $T_{\mathrm{g}}$ values of all the other bionanocomposites are decreasing.

A weak interphase can explain this effect, and the $\tan \delta$ curves obtained from a frequency sweep (Figure 5c) supported this hypothesis: the damping decreased from CNCs_lactate to CNCs_butyrate, demonstrating a reduced mobility of polymer chains caused by the CNCs/PHBV interface and corroborating a depleting interface in the case of the lactate moieties, making the stress transfer detrimental.

Finally, cryofractured samples of the bionanocomposites were observed with a SEM microscope (Figure 6). SEM analysis provided direct evidence to the morphological changes of CNCs/PHBV upon the different CNCs surface chemistry. The morphology of bionanocomposites containing esterified CNCs do not present any evidence of CNCs agglomeration (lower magnification micrographs in Figure 6), in agreement with the higher transmittance recorded by UV-vis spectroscopy, as well as their thermal and mechanical analyses. In contrast, aggregates are clearly visible in the presence of $\mathrm{CNCs} \mathrm{HCl}$ (Figure 6b), with micrometric debonding all along the agglomerate/matrix interface, consistent with a worse dispersion already demonstrated and a poor adhesion with the matrix (Figure 6 $b^{\prime}$ ).

PHBV showed a typical morphology of a brittle semicrystalline polymer (Figure 6a), with the peculiar presence of micrometric holes (Figure 6a'), as previously observed. ${ }^{27}$ In the case of CNCs_butyrate, it is difficult to distinguish individualized $\mathrm{CNCs}^{-}$from the matrix (Figure 6c). At higher magnification the interphase CNCs_butyrate/PHBV appear smooth, no pull-out is visible, and bridges connecting the matrix to the nanocrystals are noteworthy remarkable (Figure $\left.6 c^{\prime}\right)$, confirming their very good interaction with PHBV. A depleting interface with the PHBV is presented instead by the lactate decorated CNCs. Although well dispersed, this bionanocomposite showed cavities at the interfacial area surrounding the nanocrystals and CNCs pull-outs (Figure $\left.6 \mathrm{e}^{\prime}\right)$, which indicate a relatively poor interfacial adhesion caused by a lack of interactions like the unmodified CNCs. Interestingly, CNCs_but_lact brought to an intermediate interface morphology, where adhesion and debonding areas are coexisting around nanocrystals. This feature is in line with a patchy functionalized surface.

\section{CONCLUSIONS}

Modified CNCs have been successfully obtained through onepot acid hydrolysis/Fischer esterification approach, without significant changes in the aspect ratio nor affecting the crystallinity of the nanocrystals. Successful unprecedented cofunctionalization has been carried out while synthesizing the $\mathrm{CNCs}$ in the presence of two different organic acids that enable the decoration of the CNCs' surface with two functional moieties.

Bionanocomposites with the different surface-modified nanocrystals were casted from a chloroform/tetrachloroethane mixture to ensure the highest level of CNCs dispersion in the polymer matrix and, thus, to investigate the $\mathrm{CNCs} / \mathrm{PHBV}$ interface effect on the crystallization behavior of PHBV and on the efficiency of the stress transfer. The optical properties of the bionanocomposites reflected the different degrees of dispersion of modified and unmodified CNCs, already visible in the organic solvent. Surprisingly, modified-CNCs improved
PHBV films transparency, a highly important property for future applications.

Thermal and morphological analyses confirmed that CNCs surface modification impacts on PHBV crystallization behavior and on its mechanical properties. The greater effect on the bionanocomposites containing butyrate-modified CNCs, indicated the decoration of the CNCs surface with butyrate moieties as the more suitable for the use of cellulose nanocrystals as nucleating agent for the poly(3-hydroxybutyrate)-co-(3-hydroxyvaleriate).

An improved interphase between butyrate-modified nanocellulose and PHBV, is confirmed by dynamical mechanical analysis. Increased glass transition and dumping highlighted a more efficient stress transfer for this bionanocomposite, even at room temperature. These results show that the suitable surface decoration of the CNCs can hinder the PHBV mobility at the molecular scale so improving the mechanical properties of the bionanocomposite, even at very low CNCs content (2 wt \%). Our results show that green chemical modification of nanostructured cellulose is a suitable way to pave the way for broadening the applicability of PHBV bionanocomposites.

\section{ASSOCIATED CONTENT}

\section{SI Supporting Information}

The Supporting Information is available free of charge at https://pubs.acs.org/doi/10.1021/acs.biomac.9b01760.

List of acronyms and compositions of prepared bionanocomposites, XRD spectrum, deconvolution of XRD spectrum and crystallinity index $\left(\chi_{\mathrm{XRD}}\right)$ of modified and unmodified CNCs, CNCs average length $(L)$ and diameter $(D)$, and related aspect ratio $(L / D)$, TGA and relative derivative DTG curves of modified and unmodified CNCs, evolution during time of functionalized CNCs suspension in chloroform, TGA and relative DTG curves of neat PHBV and composites with 2 and 5 wt \% of CNCs_butyrate, thermal properties of neat PHBV and bionanocomposites at 2 and 5 wt \% of different type of CNCs, TGA and relative derivative DTG curves of neat PHBV and bionanocomposites with 2 wt $\%$ of modified and unmodified CNCs, Flexural storage modulus and glass transition temperature, $T_{g}$, obtained from DMTA results (PDF)

\section{AUTHOR INFORMATION}

\section{Corresponding Author}

Giada Lo Re - Department of Industrial and Materials Science, Division of Engineering Materials, Chalmers University of Technology, SE-412 96 Göteborg, Sweden; 이이.org/00000001-8840-1172; Email: giadal@chalmers.se

\section{Authors}

Chiara Magnani - Laboratory of Polymeric and Composite Materials (LPCM), Center of Innovation and Research in Materials and Polymers (CIRMAP), University of Mons (UMONS), B-7000 Mons, Belgium; 이이이.org/0000-00030858-6264

Alexander Idström - Department of Chemistry and Chemical Engineering, Division of Applied Chemistry, Chalmers University of Technology, SE-412 96 Göteborg, Sweden; (1) orcid.org/0000-0003-4765-8224

Lars Nordstierna - Department of Chemistry and Chemical Engineering, Division of Applied Chemistry, Chalmers 
University of Technology, SE-412 96 Göteborg, Sweden; Wallenberg Wood Science Center (WWSC), KTH Royal Institute of Technology, SE-100 44 Stockholm, Sweden; (1) orcid.org/0000-0002-6580-0610

Alejandro J. Müller - POLYMAT and Polymer Science and Technology Department, Faculty of Chemistry, University of the Basque Country UPV/EHU, San Sebastián 20018, Spain; IKERBASQUE, Basque Foundation for Science, Bilbao, Spain; (D) orcid.org/0000-0001-7009-7715

Philippe Dubois - Laboratory of Polymeric and Composite Materials (LPCM), Center of Innovation and Research in Materials and Polymers (CIRMAP), University of Mons (UMONS), B-7000 Mons, Belgium; 이이이.org/0000-00031534-1564

Jean-Marie Raquez - Laboratory of Polymeric and Composite Materials (LPCM), Center of Innovation and Research in Materials and Polymers (CIRMAP), University of Mons

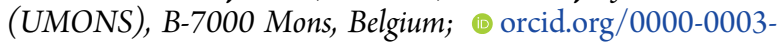
1940-7129

Complete contact information is available at:

https://pubs.acs.org/10.1021/acs.biomac.9b01760

\section{Notes}

The authors declare no competing financial interest.

\section{ACKNOWLEDGMENTS}

G.L.R. acknowledges the Chalmers Areas of Advance, Genie and KAW Biocomposites No. V-2019-0041 for financial support. Funding from the Region Wallon and European Comission in the frame of the ARC 2017-2021 Program (PHASYN Project) and from European Union's Horizon 2020 Research and Innovation Programme under the Marie Sklodowska-Curie Grant Agreement No. 778092 are gratefully acknowledged. The solid state NMR experiments were carried out at the Swedish NMR Centre in Gothenburg, Sweden.

\section{REFERENCES}

(1) Geyer, R.; Jambeck, J. R.; Law, K. L. Production, Use, and Fate of All Plastics Ever Made. Sci. Adv. 2017, 3, e1700782.

(2) Kirk, A. The shocking facts about the scale of plastic pollution choking our seas. https://www.telegraph.co.uk/news/0/shockingfacts-scale-plastic-pollution-choking-seas/ (accessed Dec 11, 2019).

(3) Ries, F. European Parliament votes for single-use plastics ban I Environment for Europeans. https://ec.europa.eu/environment/efe/ news/european-parliament-votes-single-use-plastics-ban-2019-01-18 en (accessed Dec 11, 2019).

(4) Rydz, J.; Sikorska, W.; Kyulavska, M.; Christova, D. PolyesterBased (Bio)Degradable Polymers as Environmentally Friendly Materials for Sustainable Development. Int. J. Mol. Sci. 2015, 16, 564-596.

(5) Bugnicourt, E.; Cinelli, P.; Lazzeri, A.; Alvarez, V. Polyhydroxyalkanoate (PHA): Review of Synthesis, Characteristics, Processing and Potential Applications in Packaging. eXPRESS Polym. Lett. 2014, 8, 791-808.

(6) Yu, J. In Bioprocessing for Value-Added Products from Renewable Resources, 1st ed.; Yang, S.-T., Ed.; Elsevier Science, 2007; pp 585610.

(7) Volova, T. G.; Prudnikova, S. V.; Vinogradova, O. N.; Syrvacheva, D. A.; Shishatskaya, E. I. Microbial Degradation of Polyhydroxyalkanoates with Different Chemical Compositions and Their Biodegradability. Microb. Ecol. 2017, 73, 353-367.

(8) Volova, T. G.; Boyandin, A. N.; Vasiliev, A. D.; Karpov, V. A.; Prudnikova, S. V.; Mishukova, O. V.; Boyarskikh, U. A.; Filipenko, M. L.; Rudnev, V. P.; Bá Xuân, B.; Dũng, V. V.; Gitelson, I. I. Biodegradation of Polyhydroxyalkanoates (PHAs) in Tropical Coastal
Waters and Identification of PHA-Degrading Bacteria. Polym. Degrad. Stab. 2010, 95, 2350-2359.

(9) Gilmore, D. F.; Fuller, R. C.; Schneider, B.; Lenz, R. W.; Lotti, N.; Scandola, M. Biodegradability of Blends of Poly(3-Hydroxybutyrate-Co-3-Hydroxyvalerate) with Cellulose Acetate Esters in Activated Sludge. J. Environ. Polym. Degrad. 1994, 2, 49-57.

(10) Doi, Y. Microbial Polyesters; VCH: New York, 1990.

(11) Singh, A. K.; Srivastava, J. K.; Chandel, A. K.; Sharma, L.; Mallick, N.; Singh, S. P. Biomedical Applications of Microbially Engineered Polyhydroxyalkanoates: An Insight into Recent Advances, Bottlenecks, and Solutions. Appl. Microbiol. Biotechnol. 2019, 103, 2007-2032.

(12) Lettner, M.; Schöggl, J. P.; Stern, T. Factors Influencing the Market Diffusion of Bio-Based Plastics: Results of Four Comparative Scenario Analyses. J. Cleaner Prod. 2017, 157, 289-298.

(13) Kunioka, M.; Doi, Y. Thermal Degradation of Microbial Copolyesters: Poly(3-Hydroxybutyrate-Co-3-Hydroxyvalerate) and Poly(3-Hydroxybutyrate-Co-4-Hydroxybutyrate). Macromolecules 1990, 23, 1933-1936.

(14) Kamiya, N.; Yamamoto, Y.; Inoue, Y.; Chujo, R.; Doi, Y. Microstructure of Bacterially Synthesized Poly(3-HydroxybutyrateCo-3-Hydroxyvalerate). Macromolecules 1989, 22, 1676-1682.

(15) Ferreira, B. M. P.; Zavaglia, C. A. C.; Duek, E. A. R. Films of PLLA/PHBV: Thermal, Morphological, and Mechanical Characterization. J. Appl. Polym. Sci. 2002, 86, 2898-2906.

(16) Ha, C. S.; Cho, W. J. Miscibility, Properties, and Biodegradability of Microbial Polyester Containing Blends. Prog. Polym. Sci. 2002, 27, 759-809.

(17) Corre, Y.-M.; Bruzaud, S.; Grohens, Y. Poly(3-Hydroxybutyrate-Co-3-Hydroxyvalerate) and Poly(Propylene Carbonate) Blends: An Efficient Method to Finely Adjust Properties of Functional Materials. Macromol. Mater. Eng. 2013, 298, 1176-1183.

(18) Requena, R.; Jiménez, A.; Vargas, M.; Chiralt, A. Effect of Plasticizers on Thermal and Physical Properties of CompressionMoulded Poly[(3-Hydroxybutyrate)-Co-(3-Hydroxyvalerate)] Films. Polym. Test. 2016, 56, 45-53.

(19) Jost, V.; Langowski, H. C. Effect of Different Plasticisers on the Mechanical and Barrier Properties of Extruded Cast PHBV Films. Eur. Polym. J. 2015, 68, 302-312.

(20) Kelly, C. A.; Fitzgerald, A. V. L.; Jenkins, M. J. Control of the Secondary Crystallisation Process in Poly(Hydroxybutyrate-CoHydroxyvalerate) through the Incorporation of Poly(Ethylene Glycol). Polym. Degrad. Stab. 2018, 148, 67-74.

(21) Zhu, C.; Nomura, C. T.; Perrotta, J. A.; Stipanovic, A. J.; Nakas, J. P. The Effect of Nucleating Agents on Physical Properties of Poly-3Hydroxybutyrate (PHB) and Poly-3-Hydroxybutyrate-Co-3-Hydroxyvalerate (PHB-Co-HV) Produced by Burkholderia Cepacia ATCC 17759. Polym. Test. 2012, 31, 579-585.

(22) Whitehouse, R. S.; Padwa, A. R. Nucleating Agents for Polyhydroxyalkanoates. U.S. Patent 847023, July 16, 2013.

(23) Habibi, Y.; Lucia, L. A.; Rojas, O. J. Cellulose Nanocrystals: Chemistry, Self-Assembly, and Applications. Chem. Rev. 2010, 110, 3479-3500.

(24) Ten, E.; Turtle, J.; Bahr, D.; Jiang, L.; Wolcott, M. Thermal and Mechanical Properties of Poly(3-Hydroxybutyrate-Co-3-Hydroxyvalerate)/Cellulose Nanowhiskers Composites. Polymer 2010, 51, 2652-2660.

(25) Yu, H.; Yan, C.; Yao, J. Fully Biodegradable Food Packaging Materials Based on Functionalized Cellulose Nanocrystals/Poly(3Hydroxybutyrate-Co-3-Hydroxyvalerate) Nanocomposites. RSC Adv. 2014, 4, 59792-59802.

(26) Malmir, S.; Montero, B.; Rico, M.; Barral, L.; Bouza, R.; Farrag, Y. PHBV/CNC Bionanocomposites Processed by Extrusion: Structural Characterization and Properties. Polym. Compos. 2019, 40, E275-E284.

(27) Malmir, S.; Montero, B.; Rico, M.; Barral, L.; Bouza, R. Morphology, Thermal and Barrier Properties of Biodegradable Films of Poly (3-Hydroxybutyrate-Co-3-Hydroxyvalerate) Containing Cellulose Nanocrystals. Composites, Part A 2017, 93, 41-48. 
(28) Yu, H. Y.; Qin, Z. Y.; Liu, L.; Yang, X. G.; Zhou, Y.; Yao, J. M. Comparison of the Reinforcing Effects for Cellulose Nanocrystals Obtained by Sulfuric and Hydrochloric Acid Hydrolysis on the Mechanical and Thermal Properties of Bacterial Polyester. Compos. Sci. Technol. 2013, 87, 22-28.

(29) Jun, D.; Guomin, Z.; Mingzhu, P.; Leilei, Z.; Dagang, L.; Rui, Z. Crystallization and Mechanical Properties of Reinforced PHBV Composites Using Melt Compounding: Effect of CNCs and CNFs. Carbohydr. Polym. 2017, 168, 255-262.

(30) Jiang, L.; Morelius, E.; Zhang, J.; Wolcott, M.; Holbery, J. Study of the Poly(3-Hydroxybutyrate-Co-3-Hydroxyvalerate)/Cellulose Nanowhisker Composites Prepared by Solution Casting and Melt Processing. J. Compos. Mater. 2008, 42, 2629-2645.

(31) Yu, H. Y.; Yao, J. M. Reinforcing Properties of Bacterial Polyester with Different Cellulose Nanocrystals via Modulating Hydrogen Bonds. Compos. Sci. Technol. 2016, 136, 53-60.

(32) Habibi, Y.; Goffin, A. L.; Schiltz, N.; Duquesne, E.; Dubois, P.; Dufresne, A. Bionanocomposites Based on $\operatorname{Poly}(\varepsilon$-Caprolactone)Grafted Cellulose Nanocrystals by Ring-Opening Polymerization. J. Mater. Chem. 2008, 18, 5002-5010.

(33) Habibi, Y. Key Advances in the Chemical Modification of Nanocelluloses. Chem. Soc. Rev. 2014, 43, 1519-1542.

(34) Lin, N.; Huang, J.; Chang, P. R.; Feng, J.; Yu, J. Surface Acetylation of Cellulose Nanocrystal and Its Reinforcing Function in Poly(Lactic Acid). Carbohydr. Polym. 2011, 83, 1834-1842.

(35) Yu, H. Y.; Qin, Z. Y.; Yan, C. F.; Yao, J. M. Green Nanocomposites Based on Functionalized Cellulose Nanocrystals: A Study on the Relationship between Interfacial Interaction and Property Enhancement. ACS Sustainable Chem. Eng. 2014, 2, 875886.

(36) Zhang, J.; Li, M.-C.; Zhang, X.; Ren, S.; Dong, L.; Lee, S.; Cheng, H. N.; Lei, T.; Wu, Q. Surface Modified Cellulose Nanocrystals for Tailoring Interfacial Miscibility and Microphase Separation of Polymer Nanocomposites. Cellulose 2019, 26, 43014312.

(37) Braun, B.; Dorgan, J. R. Single-Step Method for the Isolation and Surface Functionalization of Cellulosic Nanowhiskers. Biomacromolecules 2009, 10, 334-341.

(38) Spinella, S.; Lo Re, G.; Liu, B.; Dorgan, J.; Habibi, Y.; Leclère, P.; Raquez, J. M.; Dubois, P.; Gross, R. A. Polylactide/Cellulose Nanocrystal Nanocomposites: Efficient Routes for Nanofiber Modification and Effects of Nanofiber Chemistry on PLA Reinforcement. Polymer 2015, 65, 9-17.

(39) Spinella, S.; Maiorana, A.; Qian, Q.; Dawson, N. J.; Hepworth, V.; McCallum, S. A.; Ganesh, M.; Singer, K. D.; Gross, R. A. Concurrent Cellulose Hydrolysis and Esterification to Prepare Surface-Modified Cellulose Nanocrystal Decorated with Carboxylic Acid Moieties. ACS Sustainable Chem. Eng. 2016, 4, 1538-1550.

(40) Lo Re, G.; Engström, J.; Wu, Q.; Malmström, E.; Gedde, U. W.; Olsson, R. T.; Berglund, L. Improved Cellulose Nanofibril Dispersion in Melt-Processed Polycaprolactone Nanocomposites by a LatexMediated Interphase and Wet Feeding as LDPE Alternative. ACS Appl. Nano Mater. 2018, 1, 2669-2677.

(41) Dufresne, A. Cellulose Nanomaterials as Green Nanoreinforcements for Polymer Nanocomposites. Philos. Trans. R. Soc., A 2018, 376, 20170040.

(42) Oksman, K.; Aitomäki, Y.; Mathew, A. P.; Siqueira, G.; Zhou, Q.; Butylina, S.; Tanpichai, S.; Zhou, X.; Hooshmand, S. Review of the Recent Developments in Cellulose Nanocomposite Processing. Composites, Part A 2016, 83, 2-18.

(43) Park, S.; Baker, J. O.; Himmel, M. E.; Parilla, P. A.; Johnson, D. K. Cellulose Crystallinity Index: Measurement Techniques and Their Impact on Interpreting Cellulase Performance. Biotechnol. Biofuels 2010, 3, 10 .

(44) Chen, L.; Wang, Q.; Hirth, K.; Baez, C.; Agarwal, U. P.; Zhu, J. Y. Tailoring the Yield and Characteristics of Wood Cellulose Nanocrystals (CNC) Using Concentrated Acid Hydrolysis. Cellulose 2015, 22, 1753-1762.
(45) Wickholm, K.; Larsson, P. T.; Iversen, T. Assignment of NonCrystalline Forms in Cellulose I by CP/MAS 13C NMR Spectroscopy. Carbohydr. Res. 1998, 312, 123-129.

(46) Gårdebjer, S.; Bergstrand, A.; Idström, A.; Börstell, C.; Naana, S.; Nordstierna, L.; Larsson, A. Solid-State NMR to Quantify Surface Coverage and Chain Length of Lactic Acid Modified Cellulose Nanocrystals, Used as Fillers in Biodegradable Composites. Compos. Sci. Technol. 2015, 107, 1-9.

(47) Park, S.; Johnson, D. K.; Ishizawa, C. I.; Parilla, P. A.; Davis, M. F. Measuring the Crystallinity Index of Cellulose by Solid State 13C Nuclear Magnetic Resonance. Cellulose 2009, 16, 641-647.

(48) Eichhorn, S. J.; Dufresne, A.; Aranguren, M.; Marcovich, N. E.; Capadona, J. R.; Rowan, S. J.; Weder, C.; Thielemans, W.; Roman, M.; Renneckar, S.; Gindl, W.; Veigel, S.; Keckes, J.; Yano, H.; Abe, K.; Nogi, M.; Nakagaito, A. N.; Mangalam, A.; Simonsen, J.; Benight, A. S.; Bismarck, A.; Berglund, L. A.; Peijs, T. Review: Current International Research into Cellulose Nanofibres and Nanocomposites. J. Mater. Sci. 2010, 45, 1-33.

(49) Lo Re, G.; Spinella, S.; Boujemaoui, A.; Vilaseca, F.; Larsson, P. T.; Adås, F.; Berglund, L. A. Poly $(\epsilon$-Caprolactone $)$ Biocomposites Based on Acetylated Cellulose Fibers and Wet Compounding for Improved Mechanical Performance. ACS Sustainable Chem. Eng. 2018, 6, 6753-6760.

(50) Svenningsson, L.; Sparrman, T.; Bialik, E.; Bernin, D.; Nordstierna, L. Molecular Orientation Distribution of Regenerated Cellulose Fibers Investigated with Rotor Synchronized Solid State NMR Spectroscopy. Cellulose 2019, 26, 4681-4692.

(51) Moon, R. J.; Martini, A.; Nairn, J.; Simonsen, J.; Youngblood, J. Cellulose Nanomaterials Review: Structure, Properties and Nanocomposites. Chem. Soc. Rev. 2011, 40, 3941-3994.

(52) Girouard, N. M.; Xu, S.; Schueneman, G. T.; Shofner, M. L.; Meredith, J. C. Site-Selective Modification of Cellulose Nanocrystals with Isophorone Diisocyanate and Formation of Polyurethane-CNC Composites. ACS Appl. Mater. Interfaces 2016, 8, 1458-1467.

(53) Abraham, E.; Kam, D.; Nevo, Y.; Slattegard, R.; Rivkin, A.; Lapidot, S.; Shoseyov, O. Highly Modified Cellulose Nanocrystals and Formation of Epoxy-Nanocrystalline Cellulose (CNC) Nanocomposites. ACS Appl. Mater. Interfaces 2016, 8, 28086-28095.

(54) Leszczynska, A.; Radzik, P.; Szefer, E.; Mičušík, M.; Omastová, M.; Pielichowski, K. Surface Modification of Cellulose Nanocrystals with Succinic Anhydride. Polymers 2019, 11, 866.

(55) Yu, H.; Sun, B.; Zhang, D.; Chen, G.; Yang, X.; Yao, J. Reinforcement of Biodegradable Poly(3-Hydroxybutyrate-Co-3-Hydroxyvalerate) with Cellulose Nanocrystal/Silver Nanohybrids as Bifunctional Nanofillers. J. Mater. Chem. B 2014, 2, 8479-8489.

(56) Siqueira, G.; Fraschini, C.; Bras, J.; Dufresne, A.; Prud'Homme, R.; Laborie, M. P. Impact of the Nature and Shape of Cellulosic Nanoparticles on the Isothermal Crystallization Kinetics of Poly(Caprolactone). Eur. Polym. J. 2011, 47, 2216-2227.

(57) Ten, E.; Jiang, L.; Wolcott, M. P. Crystallization Kinetics of Poly(3-Hydroxybutyrate-Co-3-Hydroxyvalerate)/Cellulose Nanowhiskers Composites. Carbohydr. Polym. 2012, 90, 541-550. 